\title{
Contact Force Evaluation of Orthoses for the Treatment of Malformed Ears
}

\author{
Akihiko Hanafusa $^{1}$, Tsuneshi Isomura ${ }^{1}$, Yukio Sekiguchi ${ }^{1}$, \\ Hajime Takahashi ${ }^{2}$, and Takeyoshi Dohi ${ }^{3}$ \\ ${ }^{1}$ Department of Rehabilitation Engineering, Polytechnic University \\ 4-1-1 Hashimotodai, Sagamihara, Kanagawa 229-1196, Japan \\ hanafusa@uitec.ac.jp \\ 2 Department of Plastic Surgery, Tokyo Metropolitan Toshima Hospital \\ 33-1 Sakaemachi, Itabashi-ku, Tokyo 173-0015, Japan \\ hajime-t@toshima-hp.metro.tokyo.jp \\ ${ }^{3}$ Graduate School of Information Science and Technology, The University of Tokyo \\ 7-3-1 Hongou, Bunkyou-ku, Tokyo 113-8654, Japan \\ dohi@miki.pe.u-tokyo.ac.jp
}

\begin{abstract}
In most cases, malformed ears of neonates can be effectively treated by fitting a suitably shaped orthosis. In this study, a finite element analysis system was developed to evaluate the contact force between the orthosis and auricle. In order to reduce the contact force, two methods for modifying orthosis shape were developed. One entailed the addition of a detour curve, while the other canceled out the insertion of the nodal point where maximum force exceeded the limit during the incremental step of finite element analysis. Moreover, two small sensors were developed, one using a silicon tube and the other a strain gauge, for mounting on the orthosis and measuring contact force. Straight, curved and maximum force-restricted orthoses were manufactured for an adult auricle. Simulation and insertion experiments using silicon tube and strain gauge sensors were performed. Results demonstrated that the strength of contact force between the orthosis and auricle decreased from straight type, through curved type to the maximum force-restricted type.
\end{abstract}

\section{Introduction}

Most cases of malformed ears of neonates can be treated by mounting an appropriately shaped orthosis in the auricle [1]. Currently, the authors use orthoses made of nitinol shape memory alloy wire covered with an expanded polytetrafluoroethylene tube. Examples of both a straight orthosis mounted on cryptotia and a curved orthosis mounted on a folded helix are shown in Fig.1. We have recently developed a computer-assisted design system [2] for use in constructing the orthosis.

It is essential to control the contact force between the orthosis and auricle, to ensure that it is sufficient to correct the auricle shape, while not excessive to the degree that it may cause a decubitus-like inflammation on the auricle. This paper describes the simulation results of finite element analysis, developed using MATLAB (The Math Works Inc.), to evaluate the contact force. Moreover, methods that could potentially reduce the contact force were tested using sensors mounted on the orthosis to measure this force. 


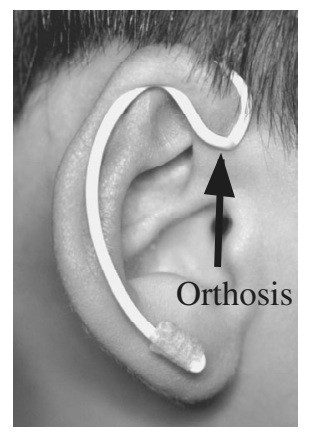

(a) Straight orthosis mounted on a cryptotia

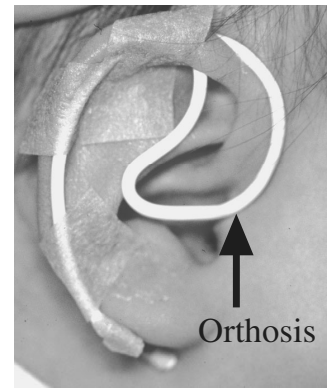

(b) Curved orthosis mounted on a folded helix

Fig. 1. Examples of orthoses made of nitinol shape memory wire

\section{Finite Element Analysis of Contact Force [3]}

\subsection{Methods}

Finite element analysis is widely used to simulate deformation or determine strength of the structure. There are numerous examples of use of this analysis in the biomedical field, including simulation of maxillofacial functions [4], and pressure at the below-knee socket. To evaluate contact force between the orthosis and auricle, one should consider the following:

- The material property of an auricle is nonlinear.

- Both auricle and orthosis deform on contact with each other.

To contend with the former concern, an incremental method was employed, whereby force or displacement was increased step by step, and moving to the next step was dependent upon a relationship between stress and strain in the current step. Displacement was increased by inserting the orthosis gradually. Moreover, a tensile test using pig's auricular cartilage was performed, and the resultant strain-stress diagram applied to the material properties.

Multiple points' constraints were also used to represent the contact deformation of auricle and orthosis. The constraint was that the orthosis nodal point never penetrated the auricle and at most on the surface of the auricular element. Using the incremental method, the constraint condition should be updated at each insertion step. In some cases, surplus constraint may occur. At the surplus constrained nodal points, the constrained force pulls the auricle inward. These constraints should be canceled and the stiffness equation should be recalculated.

A function was added that can automatically modify the orthosis shape so as to avoid excessive contact force. Briefly, we adopted a simple method that canceled the insertion of the nodal point in an incremental step where the force exceeded the limit. As the cancellation of some nodal points may change the condition of multiple points' constraint and cause excessive force at other nodal points, the condition and force should be recalculated in the insertion step. 


\subsection{Simulation Examples}

An adult male auricular model was constructed using multiple three dimensional data measured by the non-contact laser measurement system. Contours of the helix and auriculotemporal sulcus were extracted and approximated by spline using eight control points. Orthosis shape was produced by moving the third control point outward so that the top of the orthosis projected over the helix by $4 \mathrm{~mm}$. Models for finite element analysis were made under the following conditions:

- The auricle was modeled by 1093 triangular shell elements. Results of a tensile test using pig's auricular cartilage were applied to the material properties.

- The orthosis on the helix side was modeled by 33 beam elements. Results of a tensile test using nitinol shape memory wire were applied to the material properties.

- Each nodal point had six degrees of freedom.

The orthosis was inserted in the helix in 8 steps increasing by $0.5 \mathrm{~mm}$ each. The start edge of the orthosis and auriculotemporal sulcus of the auricle were clipped. Fig.2(a) shows the orthosis deformation and the force produced by the contact, and Fig.2(b) shows distribution of the contact force on the auricle. The bright area at the top of auricle and along the clipped auriculotemporal sulcus highlights where a larger force is generated. As the orthosis is inserted, it is bent backwards. The contact force between the orthosis and the auricle is concentrated in two elements. Forces at the 12th, 13 th and 14th nodal points were $0.33,0.18$ and $0.21 \mathrm{~N}$ respectively with a total force of $0.72 \mathrm{~N}$. The force at the clipped first nodal point was $0.79 \mathrm{~N}$.

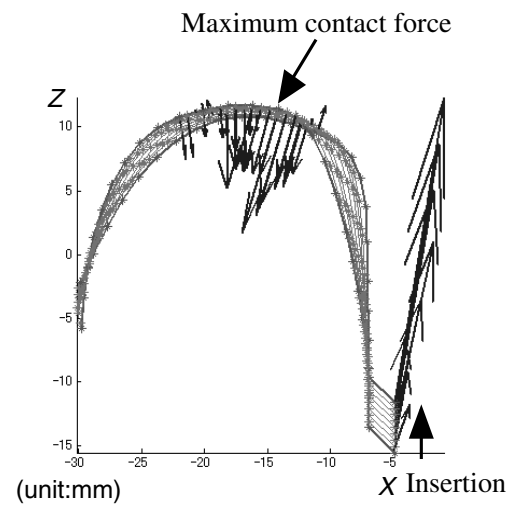

(a) Deformation of orthosis and contact force distribution.

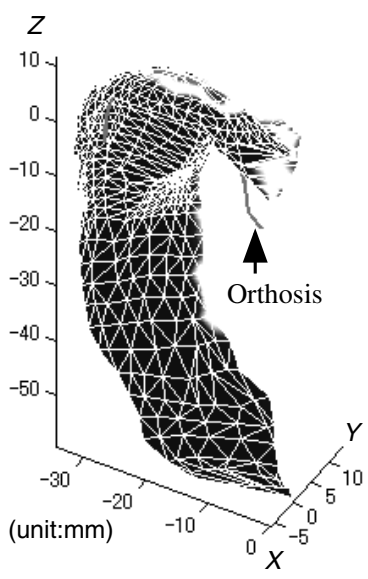

(b) Distribution of contact force on the auricle.

Fig. 2. Results of finite element analysis when the original straight type orthosis is inserted

To relax the force, two types of orthosis were constructed, a curved type and a maximum force-restricted type (Fig.3). In the former case, a detour curve was added to the start of the helix side to permit bending and thus reduce the force. With regards the maximum force-restricted type, the previously described method for automatic modification to cancel insertion of nodal points where the force exceeded the limit of $0.15 \mathrm{~N}$ was applied. 


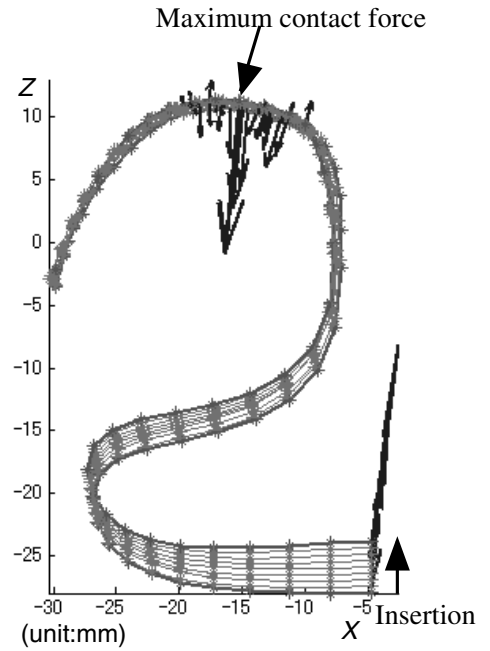

(a) Curved type

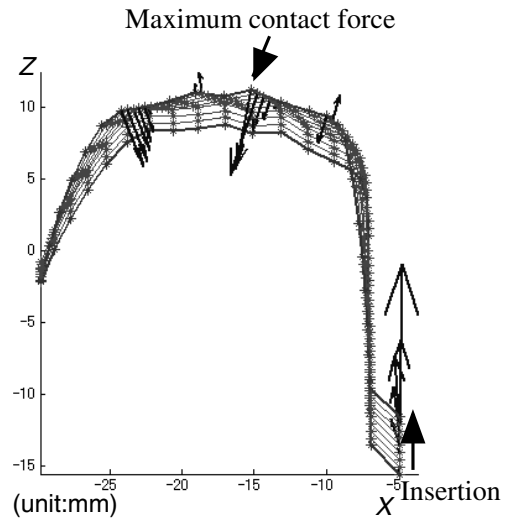

(b) Force-restricted type

Fig. 3. The deformation and contact force of finite element analysis

Fig.3 shows the deformation and contact force of finite element analysis performed under the same condition with the original straight type. With respect to the curved type, a maximum force of $0.40 \mathrm{~N}$ was generated at the two points backward in relation to the position of original shape. The maximum total force of the two elements was $0.52 \mathrm{~N}$. The force of the clipped first nodal point was also $0.52 \mathrm{~N}$. Deformation occurred mainly at the curved part, and that at the top was relatively small. With respect to the maximum force-restricted type, a maximum force of $0.18 \mathrm{~N}$ was produced at the same point as with the curved type. The backward deformation was smaller than that of the original straight type orthosis.

\section{Sensors to Measure Contact Force}

In order for a sensor to be mounted on an orthosis, it is required to be both small and thin. A sensor system was constructed using either a silicon tube or strain gauge. Straight, curved type and maximum force-restricted type orthoses were manufactured and either a silicon tube or strain gauge sensor was mounted, as illustrated in Fig.4. The sensor was positioned at the point of maximum contact force, as determined by the finite element analysis.

\subsection{Silicon Tube Sensor}

The silicon tube sensor functions based on the phenomenon that the air flow in a tube decreases when it is compressed. The inside diameter of the silicon tube was $1.0 \mathrm{~mm}$, and outside diameter $1.5 \mathrm{~mm}$. The sensor was bent over with slight tension and bonded to the orthosis. As the tube was compressed, the thickness and width of the sensor was in the order of $0.5 \mathrm{~mm}$ and $2.0 \mathrm{~mm}$ respectively. 


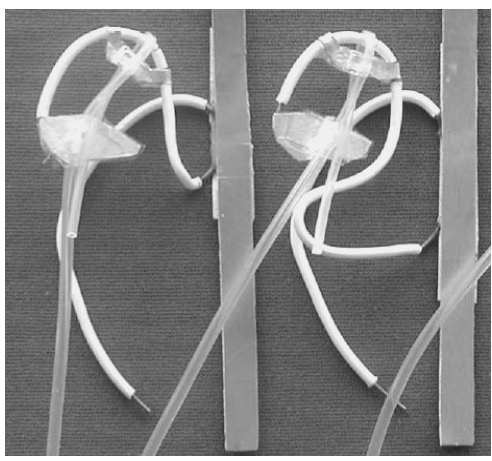

(a) Straight type (b) Curved type (Silicon tube sensor)

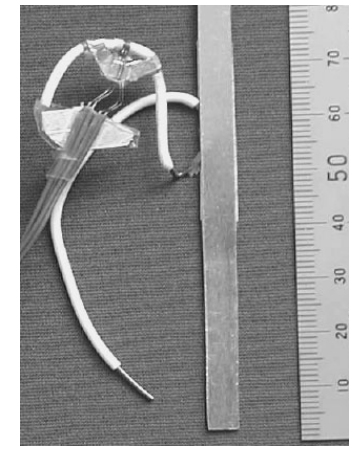

(c) Maximum force restricted type (Strain gauge sensor)

Fig. 4. Orthoses with sensors mounted

The sensor calibration system was constructed as shown in Fig.5. Firstly, air was pumped into the air tank and the valve was opened. The air flowed through the sensor and the change in pressure in the air tank was measured using the pressure sensor. The sensor part on the orthosis was compressed by the load sensor on the table. On the tip of the load sensor push rod, a $2.5 \times 2.5 \mathrm{~mm}$ silicon plate was mounted. The higher the load, the slower the air moved through the sensor and the slower the change in pressure.

The relationship between load and time constant of decreasing air pressure is illustrated in Fig.6. As load is increased, time constant also increases exponentially. The curve approximated by the quadratic equation of the natural logarithm of time constant can be used to derive the load.

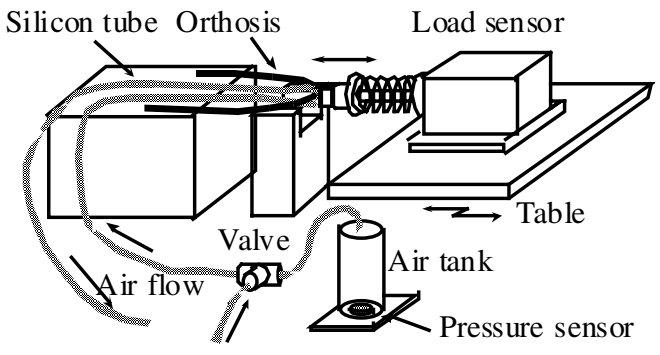

Fig. 5. Configuration of sensor calibration system

\subsection{Strain Gauge Sensor}

The strain gauge sensor functions based on the phenomenon that the gage is deformed and electric resistance is changed when compressed. The base size and grid size of the gauge were $4.2 \times 1.2 \mathrm{~mm}$ and $1.0 \times 0.68 \mathrm{~mm}$ respectively, and the thickness was $0.1 \mathrm{~mm}$. Two gauges were bonded back-to-back to limit the influence of temperature. These gauges were then bonded onto the orthosis and covered with Teflon tape of thickness $0.1 \mathrm{~mm}$. Thus, the total thickness of the sensor was $0.3 \mathrm{~mm}$. Although 
double gauges were used, coefficients of the temperature were still -0.0073 to 0.0046 $\left(\mathrm{V} /{ }^{\circ} \mathrm{C}\right)$ when there was no load. A thermistor was used to measure the temperature, and correct the sensor output voltage by subtracting the value at the same temperature as when there was no load.

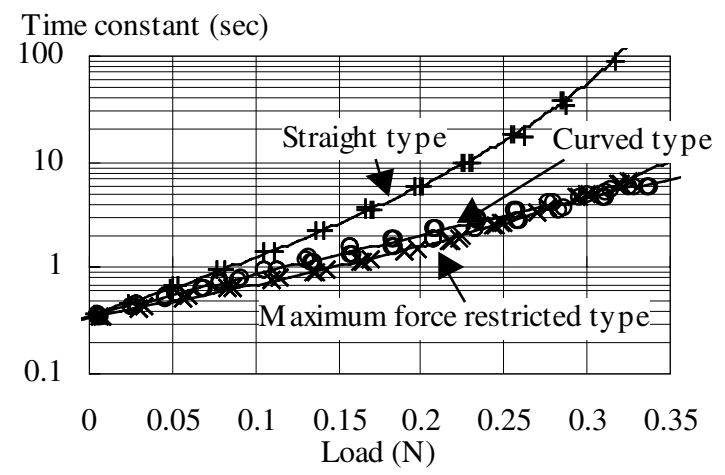

Fig. 6. Relationship between load and time constant of decreasing air pressure

Using the same calibration system illustrated in Fig.5, the relationship between load and sensor output voltage was measured. Fig.7 shows the results after temperature compensation. As the load was increased, the sensor output voltage decreased. The curve approximated by the quadratic equation of the sensor output voltage was used to derive the load.

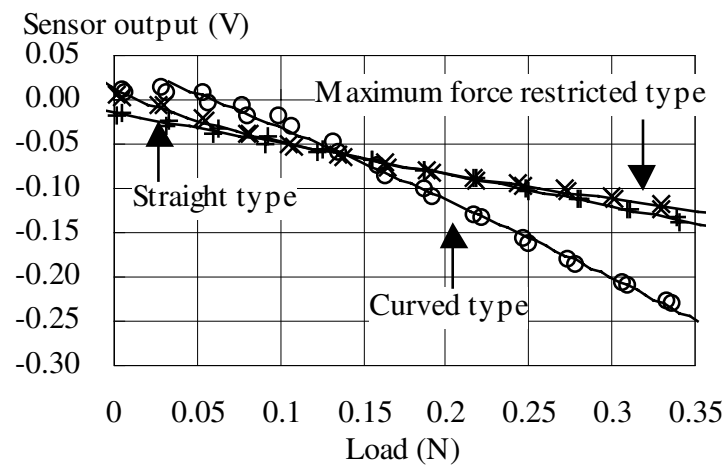

Fig. 7. Relationship between load and sensor output voltage after temperature compensation

\section{Insertion Experiment for an Adult Auricle}

An insertion test for an adult auricle, used to generate orthosis shape, was performed using six orthoses of the three previously described shapes and with two types of sensors. An aluminum plate attached to the orthosis was used to define the position on the cheek by marking the boundary of the plate. Fig.8(a) shows the results obtained using an original straight orthosis overlaid with a simulation result. Fig.8(b) shows the results obtained using a curved orthosis and (c) shows the results obtaining using a maximum force-restricted type orthosis. The overlay shows orthoses shape 
before and after insertion. The straight and almost vertical line is the boundary of the aluminum plate and the $10 \mathrm{~mm}$ square is a reference indicator. In the case of the curved orthosis, the experimental result was in close agreement with the simulation result. Using the straight or maximum force-restricted types, orthoses were bent two or three degrees extra compared to those shown in the simulation results. The reason for the difference is that the orthosis edge of the helix side was clipped in the simulation, and the edge is only pushed onto the cheek in the insertion experiment.

The insertion and removal experiment was performed 15 times and the contact force measured using the silicon tube and strain gauge sensors. Fig.9 shows the average force and range measured during the insertion experiment. The average contact forces measured by the silicon tube sensors on straight, curved and maximum forcerestricted orthoses were $0.238,0.140$ and $0.080 \mathrm{~N}$ respectively, while those measured by strain gauge sensors were $0.295,0.183$ and $0.045 \mathrm{~N}$ respectively. The difference ranged from -0.035 to $0.057 \mathrm{~N}$. As the range of values measured by the two sensors overlapped, it is estimated that the actual value is around the overlapped value. In the simulation studies, the total forces on the two elements that included the nodal point where maximum contact force was produced and the sensors were mounted were $0.72,0.52$ and $0.19 \mathrm{~N}$ respectively. The measured force corresponded to about 0.35 times that of the simulated value. To get a more accurate simulation result, a finer and more precise model that incorporates not only the auricular cartilage but also skin and muscle is necessary. Moreover, it is essential to know the material properties of the tissue. The results of the current study demonstrate that both a modified curved type and maximum force-restricted type orthoses can reduce the contact force to a greater degree than the original straight orthosis.

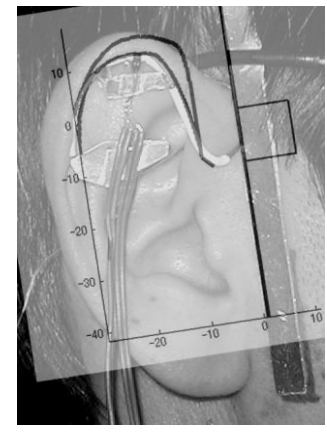

(a) Straight type

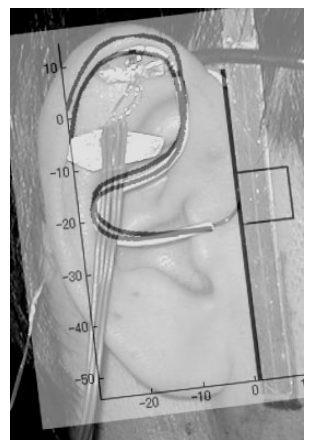

(b) Curved type

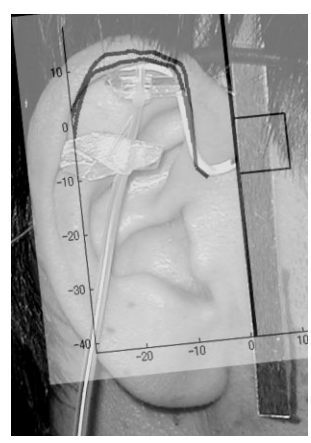

(c) Maximum force-restricted type

Fig. 8. Insertion results overlaid with simulation results

\section{Conclusion}

A finite element analysis system was developed to evaluate the contact force between the orthosis and auricle. Two methods were developed to reduce the contact force; modifying the orthosis shape by adding detour curve; and canceling the insertion of nodal point where maximum force exceeds the limit during the incremental step of finite element analysis. Also, sensors using either silicon tube or strain gauges were developed to be mounted onto an orthosis and measure the contact force. 


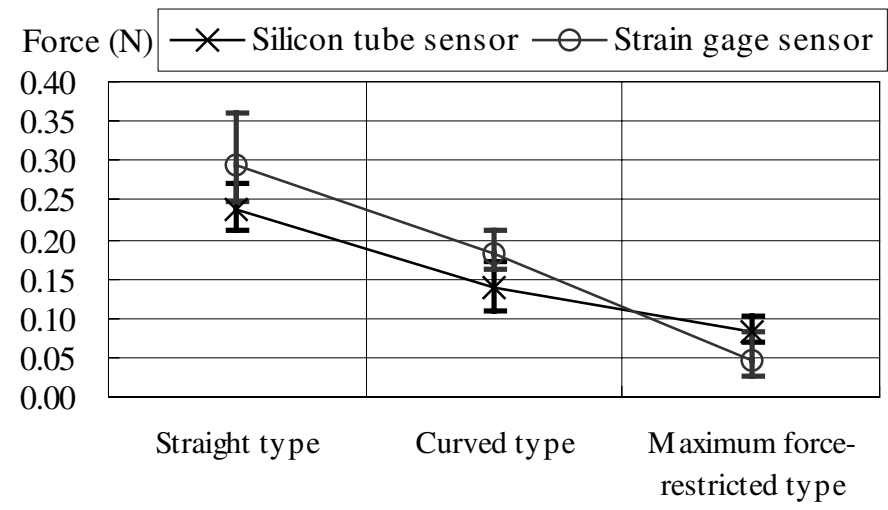

Fig. 9. Force measured during the insertion experiment for the adult auricle

Straight, curved and maximum force-restricted orthoses were made for an adult auricle. Simulation and insertion experiments using silicon tube and strain gauge sensors were performed and the results demonstrated that the strength of contact force decreased from straight type, through curved type to the maximum force-restricted type. The difference in measured contact force between the silicon tube and the strain gauge sensor ranged from -0.035 to $0.057 \mathrm{~N}$. The measured contact force corresponds to about 0.35 times that of the total simulated value along the two elements that included the nodal point where maximum contact force was produced.

\section{References}

1. Matsuo K, Hirose T, Tomono T, Iwasawa M, Katohda S, Takahashi N, Koh B : Nonsurgical Correction of Congenital Auricular Deformities in the Early Neonate, Plast. Reconstr. Surg. 73 (1984) 38-50.

2. Hanafusa A, Takahashi H, Akagi K, Isomura T : Development of Computer Assisted Orthosis Design and Manufacturing System for Malformed Ears, Computer Aided Surgery 2 (1997) 276-285.

3. Hanafusa A, Isomura T, Sekuguchi Y, Takahashi H, Dohi T: Computer Assisted Orthosis Design System for Malformed Ears -Automatic Shape Modification Method for Preventing Excessive Corrective Force-, Proc. of the World Congress on Medical Physics and Biomedical Engineering Chicago 2000 (2000) 1-3.

4. Maki K, Inou N, Mikasa M, Tanaka T, Usui T, Toki Y, Shibasaki Y: Computer-Aided Biomechanical Simulations for the Diagnosis of Maxillofacial Functions, Proc 12th International Symposium CAR and CAS (1998) 819-823. 